\title{
Efficacy of Bacillus clausii spores in the prevention of recurrent respiratory infections in children: a pilot study
}

\author{
Gian Luigi Marseglia' \\ Mariangela Tosca ${ }^{2}$ \\ Ignazio Cirillo 3 \\ Amelia Licari' \\ Maddalena Leone' \\ Alessia Marseglia' \\ Anna Maria Castellazzi ${ }^{1}$ \\ Giorgio Ciprandi ${ }^{4}$ \\ 'Dipartimento di Scienze Pediatriche, \\ Università degli Studi di Pavia, Pavia, \\ Italy; ${ }^{2}$ Centro Malattie Allergiche, \\ Istituto G. Gaslini, Genova, Italy; \\ ${ }^{3}$ Ospedale Marina Militare, La Spezia, \\ Italy; ${ }^{4}$ Semeitoica Medica I- \\ Dipartimento di Medicina Interna, \\ Azienda Ospedale-Università San \\ Martino di Genova, Genova, Italy
}

Correspondence: Giorgio Ciprandi Semeiotica Medica I - Dipartimento Medicina Interna, A.O.U. San Martino di Genova, Padiglione 3, Largo R. Benzi I0, 16132 Genova, Italy

$\mathrm{Tel}+39105552124$

Fax + 39105556682

Email gio.cip@libero.it

\begin{abstract}
Probiotic milk has been previously demonstrated to reduce the number of respiratory infections (RI) among children attending day care centres. Thus, this pilot study was aimed to assess the efficacy and the safety of 3 month treatment with Bacillus clausii in the prevention of recurrent respiratory infections (RRI) in children. Eighty children with RRI were studied: 40 of them were randomly treated with $B$. clausii for 3 months, and followed up for further 3 months; 40 were included in the control group during the same period. Children treated with B. clausii had shorter duration of RI in comparison with the control group both during the treatment phase (mean 11.7 days vs 14.37 ; $\mathrm{p}=0.037$ ) and the follow-up period (mean 6.6 days vs $10.92 ; \mathrm{p}=0.049$ ). This effect was evident also in allergic children during the follow-up. In conclusion, this pilot study provides the first preliminary evidence that $B$. clausii may exert a significant and persistent impact on RI in children and is safe and well tolerated.
\end{abstract}

Keywords: probiotic, children, respiratory infections, allergy

\section{Introduction}

Respiratory infections (RI), mainly involving the upper airways, are common in children and their recurrence constitutes a demanding challenge for the paediatrician (BWHO 1998). It has been proposed that to diagnose recurrent RI (RRI) at least one of the following criteria has to be present: 1) $>6$ RI per annum, 2) $>1$ RI per month involving the upper airways from September to April, 3) >3 RI per annum involving the lower airways (Gruppo di Studio di Immunologia della società Italiana di Pediatria 1988). Moreover, RRI represent a social problem both concerning economic aspects and the influence on the family and social milieu of the child. In addition, allergic children have more frequent and severe respiratory infections than nonallergic children (Ciprandi et al 2006). In recent years, following the increase in the incidence of antibiotic resistance, interest in preventive treatment has intensified. Such treatment should contribute to the prevention of RRI, thus reducing the usage and excessive consumption of antibiotics. Furthermore, it has been established that the intestinal microflora plays an important role in the maturation of the immune system (Holt et al 1997).

Probiotics are vital bacteria that colonize the intestine and modify its microflora with benefits for the host. They are present in foodstuffs such as yogurt and other dairy products. To exert their beneficial effects, probiotics must be able to overcome the obstacle of gastric acidity, to proliferate and to colonize the intestine even in the presence of biliary acids and of drugs such as antibiotics. They must adhere to intestinal walls, reducing their permeability and potentiate the local immune response, especially through secretory immunoglobulin (Ig) A (Isolauri et al 2001).

Probiotics are not pathogens as they are not virulent and consequently, their intake is usually not associated with undesirable effects. 
The beneficial effect of probiotics is also to be ascribed to its capacity to modulate the immune response. The immune response dependent on Th1 lymphocytes (Th1) deputy to defend from viruses and bacteria is not fully efficient because it has not completely matured. Whereas, the Th2 response to antigens prevails and is often enhanced due to contact with environmental allergens, resulting in early activation of allergic responses (type I IgE-mediated allergic responses). Probiotics are able to induce an antigenic stimulation that leads to release of interleukin (IL) 12 by antigen-presenting cells (APC), which promotes the maturation of the immune system and the Th1 response. Furthermore, they reduce the production of IL4, which specifically regulates the development of the Th2 phenotype and leads to an increase in the synthesis of $\operatorname{IgE}$, eosinophilia and atopic disease. Probiotics have anti-inflammatory properties related to the stimulation of secretory IgA synthesis in mucous membranes and the production of cytokines (Hattakka et al 2001). They also stimulate the synthesis of interferon-gamma (IFN- $\gamma$ ) and have antimicrobial activity as a result of the production of lactic acid, $\mathrm{H}_{2} \mathrm{O}_{2}$ and natural antibiotic substances with potent antiviral and antibacterial activity.

It has been previously reported that the prophylactic use of a probiotic milk reduced the number and the severity of RI among children attending day care centres (Hattakka et al 2001). Probiotics are microorganisms that exhibit a beneficial effect on the health of the host (Kaur et al 2002) and the interaction between probiotics and host has a deep impact in many ways. One is to stimulate the immune system by promoting Th1 and T regulatory (Treg) immunity, and by decreasing Th2 activity in allergic subjects (Elson and Cong 2002). In this regard, Bacillus clausii is a probiotic capable of modulating the immune response (Ciprandi et al 2005a, 2005b). Specifically, it has been demonstrated that B. clausii stimulates Th1 and Treg immunity, promoting IL12 , IFN- $\gamma$, IL-10, and transforming growth factor-beta (TGF$\beta)$ synthesis, and down-regulates Th2 response, inhibiting IL-4 production, in allergic children with RRI (Ciprandi et al 2004).

Therefore, this pilot study was performed to assess the efficacy and the safety of B. clausii in the prevention of RRI in children.

\section{Methods}

\section{Probiotic}

Bacillus clausii is an aerobic, spore-forming bacterium that is able to survive transit through the acidic environment of the stomach and colonize the intestine even in the presence of antibiotics (Duc et al 2004). B. clausii is available for oral use as a suspension of 2 billion spores per $5 \mathrm{~mL}$.

\section{Population and eligibility criteria}

Eighty children (39 males and 41 females, mean age $4.3 \pm$ 1.5 years) with RRI were enrolled in the study, among these the atopics were 18 in the $B$. clausii group and 19 in the other one. Inclusion criteria were: out-patients of both sexes, aged 3-6 years, attending a nursery or primary school, positive history for RRI in the last 12 months ( $\geq 6$ episodes), good general conditions, and written informed consent of at least one parent. Exclusion criteria were: primary or acquired immunodeficiency, hypersensitivity to the study treatment, previous (last 3 months) or concomitant administration of drugs capable of interfering with the study (eg, immunomodulants, homeopathic therapy, or systemic corticosteroids for at least 2 consecutive weeks), use of antiallergic drugs (ie, topical corticosteroids, antileukotrienes, cromones) during the study, history of chronic disease, cancer, or congenital malformation of the airways.

\section{Study design}

A randomized, single-blind, multi-centre, two arm parallelgroup protocol was designed. The study was approved by the ethics committee - IRCCS San Matteo - Pavia.

\section{Study medication}

At the first visit, $B$. clausii was randomly prescribed to 40 children in a blinded manner so that investigators were unaware which children were given the probiotic. One vial of $B$. clausii was to be taken orally twice daily for 90 days. As rescue medication for allergic children, an antihistamine (desloratadine) was allowed for symtomatic use alone.

\section{Safety}

Safety and tolerability of the probiotic were evaluated on the basis of the number and type of adverse events recorded according to the principles of good clinical practice.

\section{Study procedures}

The children were examined at study entry and every month for 3 months (treatment period). After the initial treatment period there was a further 3 months of follow-up during which the children were monitored monthly. The study 
started in January 2004 and ended in July 2004. At each visit, a history of infections occurring during the preceding period was taken and a clinical examination was also conducted. The investigators diagnosed RI on the basis of the symptoms recorded by the parent in the study diary and noted the duration of the episodes. The diagnosis of RI was made when at least 2 symptoms or fever (axillary temperature $\geq 38^{\circ} \mathrm{C}$ ), in addition to one other symptom, were present for at least 48 hours. The symptoms taken into consideration for diagnostic purposes were: mucopurulent rhinorrhea, stuffy or dripping nose or both, sore-throat, cough (dry or productive), otalgia (earache), fever, dyspnoea, and mucopurulent secretion. Allergy was diagnosed on the basis of positivity to skin prick test according to validated criteria (Ciprandi et al 2004). Eighteen allergic children were actively treated and nineteen acted as controls.

\section{Statistical analysis}

The main descriptive statistics were calculated for each variable: mean, median, and standard deviation for quantitative variables. Also the $95 \%$ confidence limits were calculated whenever relevant. The efficacy analysis was carried out using the $\chi^{2}$ test.

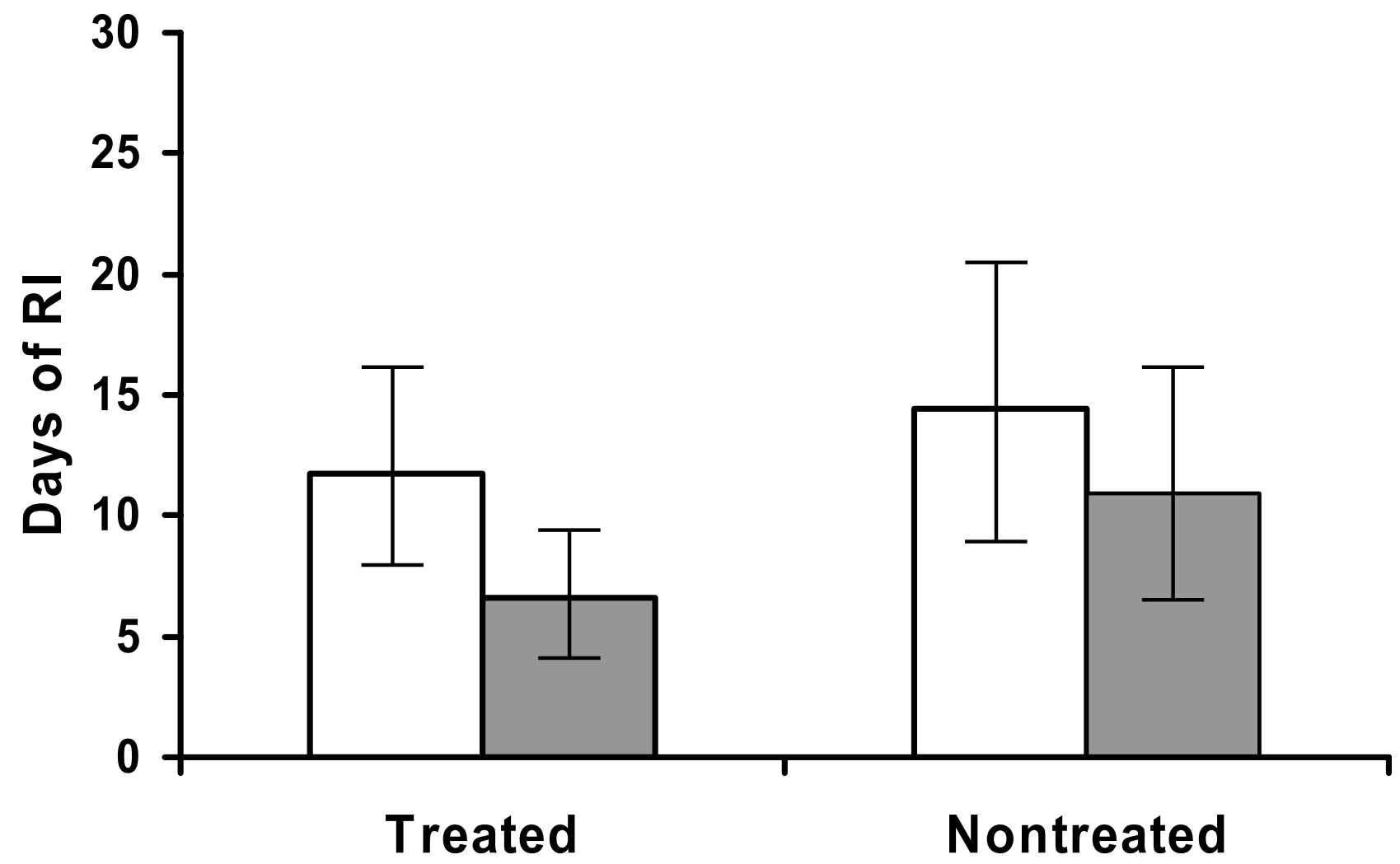

Figure I Duration of RI (expressed as days in treated and nontreated as children with RRI during the treatment period (white column) and the follow-up (grey column).

Abbreviations: RI, respiratory infections; RRI, recurrent respiratory infections.

\section{Results}

A total of 80 subjects were enrolled in the study. Forty children were treated with $B$. clausii and 40 children were in the control group. They all completed the study. different among the two groups.

\section{Efficacy}

\section{Treatment period}

Children treated with probiotic showed a significantly reduced duration (mean $11.7 \pm 4.8$ days) of RI in comparison with the control group (mean $14.37 \pm 6.3)(\mathrm{p}=0.037)$ as reported in Figure 1. The number of RI was less in the probiotic group $(3.2 \pm 0.8)$ compared with controls $(3.9 \pm$ $0.8)$. Regarding allergic children, the duration of RI was shorter in the probiotic group $(13.44 \pm 4.6)$ than in the nontreated controls $(15.36 \pm 7.4)$, as illustrated in Figure 2.

\section{Follow-up period}

Bacillus clausii-treated children had a shorter duration (6.6 $\pm 3.1)$ of RI than controls $(10.92 \pm 5)(\mathrm{p}=0.049)$. The number of RI was less in actively treated children $(1.95 \pm 0.9)$ than in controls $(3 \pm 1)$. Moreover, allergic treated children had shorter durations of RI $(7.72 \pm 2.9)$ than allergic nontreated Demographic and baseline variables were not significantly 


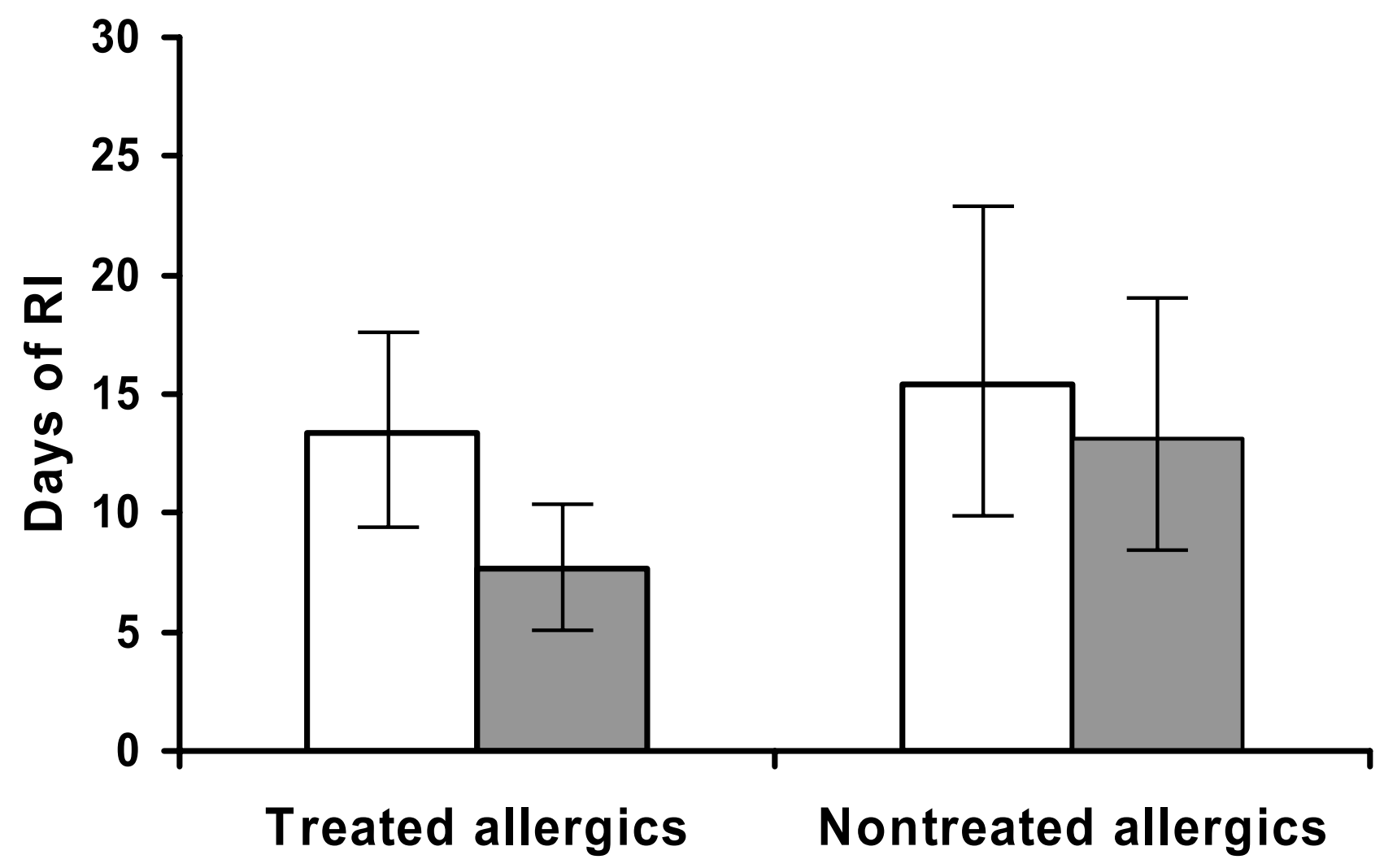

Figure 2 Duration of RI (expressed as days) in treated and nontreated allergic children with RRI during the treatment period (white column) and the follow-up (grey column).

Abbreviations: RI, respiratory infections; RRI, recurrent respiratory infections.

$(13.1 \pm 5.3)(\mathrm{p}=0.039)$. The use of symptomatic antihistamine was very slight in both groups.

\section{Safety}

None of the children were withdrawn from the study because of adverse events and very few mild adverse events occurred (3 diarrhoea episodes). Indeed, such events were either related to the underlying disease (RI) or not considered treatment-related.

The tolerability profile exhibited in the $B$. clausii group was similar to that of the control group. The proportion of patients who experienced adverse events was similar in the two groups both during the treatment phase and the follow-up.

\section{Discussion}

This preliminary study shows that $B$. clausii treatment is significantly effective $(\mathrm{p}=0.037)$ in reducing the duration of RI in children with recurrent disease. It is important to note that this study provides the first evidence concerning the capability of $B$. clausii in reducing the severity of recurrent RI. Moreover, in probiotic-treated children, the duration of RI episodes during the follow-up period was also significantly shortened $(\mathrm{p}=0.049)$. Furthemore, it is well known that allergic children experience more numerous and more severe RI than normal subjects, and yet $B$. clausii was also effective in preventing RI also in such atopic children during follow-up.

Enterogermina 2 billions $/ 5 \mathrm{ml}$ is a treatment product for oral use that contains a single active ingredient, namely polyantibiotic-resistant spores of $B$. clausii. It is provided in the form of a suspension containing 2 billion spores per $5 \mathrm{ml}$. The spores belong to 4 strains $(\mathrm{O} / \mathrm{C}, \mathrm{T}, \mathrm{N} / \mathrm{R}$, and $\mathrm{SIN})$ that are resistant to different classes of antibiotics. B. clausii is an aerobic, spore-forming bacterium that is able to inhibit the growth of pathogens in the gastrointestinal tract via three distinct mechanisms: colonization of free ecological niches, which are no longer available for the growth of other microorganisms; competition for epithelial cell adhesion, which is particularly relevant for spores in the initial or intermediate germination phase; production of antibiotics and/or enzymes secreted into the intestinal environment, especially peptide antibiotics, which are mainly active on Gram-positive bacteria, but also enzymes that exhibit lytic activity against Pseudomonas aeruginosa. The B. clausii of Enterogermina has proven to be highly resistant to gastric acidity and most antibiotics, with the exception of sulfonamides and 
trimethoprim, a few aminoglycosides and nitrofurans, combinations of penicillins with beta-lactamase inhibitors and vancomycin.

The possible mechanism of action may be due to its modulatory activity on the immune response as previously reported in a study in allergic children with RRI (Ciprandi et al 2004). In fact, RRI may be linked to a "relative" immaturity of the immune system that is typically Th2 polarized in first and second years of infancy (Ciprandi et al 2004). B. clausii could redirect the immune response towards a more physiologic Th1 oriented polarization that can adequately fight infections mainly through IFN- $\gamma$. This phenomenon is associated with on the stimulation of Treg cells releasing cytokines necessary for stimulating the Th1 response. Moreover, previous studies have demonstrated that $B$. clausii stimulates the production of IgA (Fiorini et al 1985). IgA synthesis is under the strict control of TGF- $\beta$ that is produced by Treg cells (Sporn and Roberts 1992). Therefore, different immunological mechanisms of action may explain the preventive activity exerted by $B$. clausii in reducing the duration of RI, even though immunological parameters were not investigated in this study.

In conclusion, this study provides the first preliminary evidence that $B$. clausii may exert a significant and persistent preventive activity on RI in children and is safe and well tolerated.

\section{References}

[BWHO] Bull World Health Organ. 1998. Acute respiratory infections: the forgotten pandemic. Bull World Health Organ, 76:101-3.

Ciprandi G, Tosca MA, Milanese M, et al. 2004. Cytokines evaluation in nasal lavage of allergic children after bacillus clausii administration: a pilot study. Ped Allergy Immunol, 15:148-51.

Ciprandi G, Vizzaccaro A, Cirillo I, et al. 2005a. Bacillus clausii effects in children with allergic rhinitis. Allergy, 60:702-3.

Ciprandi G, Vizzaccaro A, Cirillo I, et al. 2005b. Bacillus clausii exerts immuno-modulatory activity in allergic subjects: a pilot study. Eur Ann Allergy Clin Immunol, 37:129-33.

Ciprandi G, Tosca MA, Fasce L. 2006. Allergic children have more numerous and severe respiratory infections than non-allergic children. Ped Allergy Immunol, 17:389-91.

Duc LH, Hong HA, Barbosa TM, et al. 2004. Characterization of bacillus probiotics available for human use. Appl Env Microbiology, 70:216171.

Elson CO, Cong Y. 2002. Understanding Immune-microbial homeostasis in intestine. Immunol Res, 26:87-94.

Fiorini G, Cimminiello C, Chianese R. 1985. Bacillus subtilis selectively stimulates the synthesis of membrane bound and secreted IgA Chemioterapia, 4:310-12.

Gruppo di studio di Immunologia della società Italiana di Pediatria. 1988 Le infezioni ricorrenti nel bambino: definizione ed approccio diagnostico. Riv Immunol Allergol Pediatrica, 2:127-34.

Hatakka K, Savilahti E, Ponka A. 2001. Effect of long term consumption of probiotic milk on infections in children attending day care centers: double blind, randomised trial. $B M J, 322: 1-5$.

Holt PG, Sly PD, Bjorksten B. 1997. Atopic versus infectious diseases in childhood: a question of balance? Pediatr Allergy Immunol, 8:53-8.

Isolauri E, Sutas Y, Kankaanpaa P, et al. 2001. Probiotics: effects on immunity. Am J Clin Nutr, 73:444s-450s.

Kaur IP, Chopra K, Saini A. 2002. Probiotics: potential pharmaceutical applications. Eur J Pharm Sci, 15:1-9.

Sporn MB, Roberts AB. 1992. Transforming growth factor-beta recent progress and new challenges. J Cell Biol, 119:1017-21. 
